\title{
Mycobacterium marinum
}

National Cancer Institute

\section{Source}

National Cancer Institute. Mycobacterium marinum. NCI Thesaurus. Code C86545.

A species of aerobic, Gram positive, rod shaped bacterium assigned to the phylum

Actinobacteria. This species is acid fast, nonmotile with optimum growth on LowensteinJensen medium, urease and catalase positive, niacin and nitrate negative, and produces pigment when cultured and exposed to light. M. marinum is commonly found in bodies of fresh or saltwater and skin infections in humans are usually acquired from contact with aquariums or fish. 